\title{
PRÁCticas CULINARIAS Y (RE)CONOCIMIENTO DE LA DIVERSIDAD LOCAL DE Verduras silvestres en el Colectivo Mujeres y Maíz DE TeOpisca, Chiapas, MéXico
}

\author{
Culinary Practices in the (re)Knowledge of the Local Diversity of Wild Vegetables in Teopisca, Chiapas, \\ Mexico, Mujeres y Maíz Collective \\ Celina Guadalupe Solís-Becerra \\ Erin Ingrid Jane Estrada-Lugo
}

Resumen: Se analizan los saberes y acciones relacionados con la diversidad local de verduras silvestres, reconocida por las integrantes del Colectivo Mujeres y Maíz de Teopisca, Chiapas, México, a partir de sus prácticas culinarias. A través de métodos cualitativos y colectas botánicas se documentaron treceespecies deverduras silvestres mayormente presentesen traspatios y 33 recetas culinarias con incorporación de tales especies. Se destaca la importancia de las verduras silvestres, la gastronomía local, el trabajo cotidiano y la vida campesina, con los cuales se enriquece la relación que las mujeres del colectivo y sus familias tienen con la biodiversidad local a través de su cultura alimentaria.

Palabras clave: verduras olvidadas, cultura alimentaria, sabiduría local, gastronomía local, huerto familiar.

Abstract: This work analyzes the knowledge and actions related to wild vegetables local diversity recognized by Mujeres y MaizCollective's members from Teopisca, Chiapas, México, through their culinary practices. Through qualitative methods and botanical collections, thirteen species of wild vegetables were documented, mostly present in home gardens and 33 recipes incorporating these species. It highlights the importance of wild vegetables, local cuisine, daily work and rural way of living in enriching the relationship between women belonging to the Collective's and their families with the local biodiversity through its food culture.

Keywords: forgotten vegetables, food culture, local wisdom, local gastronomy, home garden.

Celina Guadalupe Solís Becerra, maestra en ciencias en recursos naturales y desarrollo rural por El Colegio de la Frontera Sur. Catedrática de la Universidad Intercultural de Chiapas, México. Temas de especialización: antropología de la alimentación, relación sociedad-naturaleza, ecología cultural. Correo electrónico: tarumba00@gmail.com.

Erin Ingrid Jane Estrada Lugo, doctora en antropología social por la Universidad Iberoamericana. Investigadora en El Colegio de la Frontera Sur, México. Temas de especialización: grupo doméstico y parentesco, derecho consuetudinario maya, organización social y sistemas agrícolas tradicionales. Correo electrónico: eestrada@ecosur.mx.
Enviado a dictamen: 22 de enero de 2014. Aprobación: 21 de marzo de 2014.

Revisiones: 1. 


\section{Introducción}

U no de los ámbitos donde se relacionan de forma más evidente el ser humano y la naturaleza es la alimentación humana. Cada comida incorpora diferentes especies comestibles que varían de una a otra cultura (Castro y Balzaretti, 2003; Fernández-Armesto, 2004). Sin embargo, hoy existe una tendencia global hacia la estandarización alimentaria favorecida por el modelo de desarrollo económico neoliberal (Rodríguez, 2007; Nájera y Álvarez, 2010) y la producción y aprovechamiento de un reducido número de especies alimenticias (Díaz y Gómez, 2001; Diamond, 2007; Boege, 2008; New Agriculturalist, 2008). De las cincuenta mil especies vegetales potencialmente comestibles del planeta, la población mundial basa su dieta en el cultivo de tres cereales: trigo, arroz y maíz (Loftas, 1995). Esto significa que existe un porcentaje amplio de especies comestibles locales y no comerciales que no es tomado en cuenta por los modelos de alimentación, producción o comercialización dominantes que ejercen una influencia estandarizante en nuestros hábitos y preferencias de consumo (González, 2008; New Agriculturalist, 2008). Dichos modelos se valen de la regulación del mercado y de la producción global de alimentos a través del control ejercido por contadas empresas agroindustriales (Toledo et al., 1985; Fernández-Armesto, 2004; Langreo, 2008). Tal situación incrementa la dependencia de los pequeños productores hacia insumos comerciales, como los agroquímicos, con la cual se encarece la producción, se reduce el poder adquisitivo, se dificulta la recuperación de los costos de inversión y se limita el ejercicio de la soberanía alimentaria de los productores (Toledo et al., 1985; Rosset, 2007; Boege, 2008); además, se pierde diversidad por dicha forma de producción industrial (Altieri y Nicholls, 2005; Fernández-Armesto, 2004). Asimismo, las grandes cadenas de supermercados imponen precios y acaparan el abasto alimentario en los centros urbanos del mundo, de modo que fomentan un distanciamiento entre los productores primarios y los consumidores finales, así como la desvinculación entre la población urbana y los ecosistemas de los que ésta depende para subsistir (Castro y Balzaretti, 2003; Fernández-Armesto, 2004).

Este contexto, en el que se reduce el conocimiento de los ecosistemas locales y sus especies comestibles, propicia en varios casos el deterioro de los saberes y las prácticas culturales de los consumidores, lo que puede conducir a que se ignore la biodiversidad local a través de la supresión de especies locales de la dieta y la cocina de un lugar específico (Castro y Balzaretti, 2003; González, 2008). En su estudio, Castro y Balzaretti (2003) registraron, en la ciudad de Guadalajara, México, que del $100 \%(n=165)$ de las especies alimentarias consumidas en el siglo XVI, sólo el 13.3\% (n=22) continúa ingiriéndose en la actualidad. Las autoras sugieren que el desuso del $86.7 \%$ de la riqueza biológica de especies comestibles en la región, reduce la sensibilidad de la ciudadanía ante los casos en que una o varias de tales especies se encuentren amenazadas o formen parte de un ecosistema en proceso de destrucción. Dada la estrecha relación que guarda la cultura alimentaria con el conocimiento de la biodiversidad y los ecosistemas locales, en este estudio se consideran las prácticas culinarias como medio para objetivar acciones y saberes locales asociados a especies comestibles olvidadas o poco valoradas, a fin de que se asimile y difunda la importancia de esta biodiversidad y se afronten problemas derivados de su olvido o su poca valoración (Castro y Balzaretti, 2003; Linares et al., 2006).

Especies comestibles poco valoradas son las verduras silvestres, las cuales forman parte de la dieta y de las tradiciones culinarias de distintas sociedades desde hace cientos de años (Heiser, 1985; Bye y Linares, 2000; Van den Eynden y Cueva, 2008). En América, desde la época precolombina se reconoció el valor nutricional de estas plantas (Heiser, 1985). Otros estudios subrayan la trascendencia de la diversificación del consumo de plantas silvestres y cultivadas para la salud humana (Bélanger y Johs, 2008,) así como el destacado papel del conocimiento etnobotánico en la salud de las nuevas generaciones (McDade et al., 2007).

En las zonas del norte, occidente y centro de la República mexicana, se conoce como "quelites" a las 
verduras silvestres que crecen dentro de la milpa y en huertos familiares (Bye y Linares, 2000; Resina, 2010). En virtud de que surgen en las zonas de cultivo sin ser sembradas, las verduras silvestres pueden considerarse malezas, por lo que su subsistencia se ve obstaculizada por el uso de herbicidas (Bye et al., 2008; Ramírez-Salinas y Castro-Ramírez, 2011, Vibrans 2012). Diversos autores señalan que desde la época colonial ha habido variaciones en el conocimiento y uso de estas verduras (Bye y Linares, 2000; Mera et al., 2005). Mientras que en las primeras décadas de dicho período, en el Valle de México, se documentaron entre 84 y 150 especies de quelites, hoy en día sólo se consumen quince de ellas, lo que representa entre el $17.8 \%$ y el $10 \%$ de la riqueza inicialmente reportada (Bye y Linares, 2000).

Generalmente, las verduras silvestres no constituyen alimentos comerciales ni altamente productivos. Su empleo en la dieta cotidiana tiende a reducirse o sustituirse por otras verduras de mayor distribución, consideradas de mayor prestigio, como las acelgas, el repollo o las espinacas (Bye y Linares, 2000; De Garine y Vargas, 2006; González, 2008). La recuperación de alimentos locales poco valorados como las verduras silvestres es una alternativa ante la homogeneización alimentaria por su valor nutricional, cultural y biológico (Linares et al, 2006; González, 2008; New Agriculturalist, 2008) y, por ende, para la superación de las crisis ambiental y alimentaria.

El Colectivo Mujeres y Maíz está liderado por mujeres y constituidopor grupos familiares de la región Altos de Chiapas, México. Elabora productos a base de variedades locales de maíz como estrategia comercial y de rescate de dichas variedades; promueve el derecho de estas mujeres y de su comunidad a elegir lo que comen y la manera en que lo producen; y prioriza la producción y el consumo local de alimentos. El objetivo de este trabajo es analizar las acciones y saberes, relacionados con la diversidad local de verduras silvestres, que tienen las integrantes del Colectivo Mujeres y Maíz de Teopisca, Chiapas, a través de sus prácticas culinarias. Los resultados proporcionan elementos para la construcción de alternativas localmente apropiadas frente a la crisis ambiental y al proceso global de homogeneización alimentaria.

\section{Descripción del grupo y área de estudio}

El Colectivo Mujeres y Maíz se conformó en el año 2008. Sus integrantes actuales habitan en San Cristóbal de Las Casas, Teopisca y Amatenango del Valle, Chiapas, México, donde han conformado un subgrupo por localidad. En cada subgrupo las integrantes mantienen lazos de parentesco entre ellas. Se trata en su mayoría de madres de familia que cuentan o no con el apoyo de sus parejas, quienes trabajan en el campo, la construcción y otros empleos generalmente eventuales. En el colectivo, las mujeres elaboran y venden productos a base de variedades locales de maíz, con lo que su economía familiar se favorece, a la vez que se fomenta una alimentación saludable mediante el consumo de alimentos producidos localmente, tanto en sus hogares, como en los de los consumidores de la ciudad de San Cristóbal de Las Casas (Mujeres y Maíz, 2010). Dado que esta investigación se refiere a las verduras silvestres asociadas a espacios de cultivo, sólo se tomará en cuenta a las mujeres del colectivo que radican en Teopisca, población reconocida en la región por su productividad agrícola (INAFED, 2005), en contraposición a la dificultad de acceso a la tierra y a la poca extensión de los espacios de cultivo existentes en San Cristóbal de Las Casas (Flores, 2012). Las mujeres de Amatenango del Valle no fueron incluidas debido a su reciente incorporación, en 2011, al colectivo "Mujeres y Maíz".

El Colectivo Mujeres y Maíz de Teopisca está integrado por doce mujeres, que tienen entre 18 y 55 años de edad, quienes pertenecen a seis grupos familiares distintos. El 58.3\% de estas mujeres y sus parejas cuenta con una parcela para el cultivo de maíz, mientras que el 100\% de ellas tiene acceso al huerto familiar de la unidad doméstica en la que habita. Para cumplir con sus actividades, estas mujeres se organizan en grupos de dos a cuatro integrantes que acuden regularmente a la ciudad de San Cristóbal de Las Casas para distribuir sus productos en centros educativos, en el comedor comunitario La Milpa y en los espacios de la Red de 
Productores y Consumidores Responsables Comida Sana y Cercana, a través del tianguis del mismo nombre (Mujeres y Maíz, 2010; RPCRCSC, 2012).

La población de Teopisca es cabecera del municipio del mismo nombre y pertenece a la región socioeconómica Altos tsotsil-tseltal del estado de Chiapas (INEGI, 2010). Se ubica entre las coordenadas geográficas $16^{\circ} 32$ '22" Norte y $92^{\circ} 28^{\prime} 25^{\prime \prime}$ Oeste, a una altitud de 1780 metros sobre el nivel del mar (mapa 1). El municipio se encuentra en una zona de transición tanto cultural como climática. El noreste, conocido popularmente como "tierra fría", es de clima templado subhúmedo con lluvias en verano, mientras que el suroeste, llamado "tierra caliente", posee un clima semicálido subhúmedo con lluvias en verano (CEIEG, 2010). La precipitación media anual va de 1000 a 1300 milímetros y la temperatura media anual es de 12 a $18^{\circ} \mathrm{C}$. La vegetación corresponde a bosques de coníferas, bosques de encino y vegetación inducida (CEIEG, 2010). La ciudad cuenta con 16,240 habitantes (INEGI, 2010). Aunque se ubica en una zona geográfica predominantemente indígena, la mayor parte de la población urbana es mestiza. Las principales actividades económicas son la agricultura de temporal, la ganadería, el aprovechamiento forestal, el envasado artesanal de palmito y la mano de obra para la construcción (Gobierno Municipal de Teopisca, 1984).

\section{Estrategia metodológica}

El trabajo de campo se realizó de febrero a agosto de 2012. Para documentar datos personales y el papel que juega cada integrante dentro del colectivo, en Teopisca, se aplicó una encuesta por miembro. Posteriormente se seleccionaron tres mujeres jefas de familia pertenecientes a grupos familiares distintos, elegidas por su papel dentro del colectivo, la calidad de la información proporcionada y el reconocimiento por parte de sus compañeras como conocedoras del tema. Se aplicaron tres entrevistas semiestructuradas a las mujeres seleccionadas y sus grupos familiares. Cada entrevista consistió en un día completo de visita e interacción con el grupo familiar, combinada con observación participante para ampliar la información sobre especies de verduras silvestres conocidas, lugares y formas de abastecimiento, preparación y consumo, preferencias familiares en torno a su ingesta y recetas culinarias asociadas a dichas especies. Siempre que fue posible, las entrevistas se grabaron en audio. La distribución natural de la verdura silvestre principal empleada en cada receta, la procedencia de la técnica de preparación principal, así como la presencia de utensilios, procedimientos $u$ otros ingredientes distintivos de la gastronomía tradicional mexicana (Barros y Buenrostro, 2002; Crosby, 2003; De'Angeli y De'Angeli, 2007), fueron los criterios para determinar el posible arraigo de cada platillo reportado. Para documentar la diversidad de verduras silvestres conocidas y consumidas se tomaron fotografías y se colectaron muestras botánicas para su identificación taxonómica tanto en los huertos familiares y parcelas de las mujeres, como en las visitas mensuales realizadas al mercado y al supermercado locales. Las especies de hierbas comestibles no colectadas se identificaron a través de las descripciones proporcionadas por las mujeres y mediante consultas a expertos y literatura especializada. Los ejemplares están depositados en el Herbario del Colegio de la Frontera Sur, en la unidad de San Cristóbal de Las Casas. Para triangular y complementar la información se culminó con un taller participativo con todas las integrantes del Colectivo Mujeres y Maíz de Teopisca. El análisis de las entrevistas se efectuó a través de la categorización del material discursivo derivado de las grabaciones y notas de campo, así como del cotejo de información proporcionada por cada entrevistada.

\section{Resultados}

En cuanto a la diversidad local de verduras silvestres en las prácticas culinarias del Colectivo Mujeres y Maíz de Teopisca, en el cuadro 1 se reporta la riqueza de verduras silvestres referidas por las entrevistadas. Fueron identificadas trece especies distribuidas en nueve familias; destacan la Brassicaceae - tres especies - la Asteraceae y la Chenopodiaceae - dos especies cada una-. 
El 62\% (n=8) de estas especies es nativo de la zona de estudio y el $38 \%(n=5)$ se trata de especies introducidas.

Las prácticas culinarias se refieren a los materiales involucrados, las técnicas empleadas y las acciones realizadas durante las fases de obtención, preparación, conservación y consumo de los alimentos y se componen de tres fases (Meléndez y Cañez, 2010; Nájera y Álvarez, 2010) que se examinan a continuación:

\section{Abastecimiento}

Las especies de verduras silvestres (cuadro 1) se encontraron en distintos lugares como huertos familiares, el mercado local, caminos y calles dentro de la localidad, milpas anexas a los domicilios visitados y parcelas de cultivo ubicadas en "tierra caliente". El 92.3\% $(n=12)$ se encontró tanto en traspatios, como en otros lugares de colecta, el $61.5 \%(\mathrm{n}=8)$ se puede obtener en el mercado local, lo que contrasta con que ninguna de las especies se halló durante los meses de monitoreo en la sucursal de la cadena Wal-Mart que opera en Teopisca desde julio de 2011. El 53.8\% se encontró en los caminos y calles; y el 38.5\%, en las milpas. Otras especies de verduras silvestres comestibles son más comunes en hábitats específicos conocidos localmente como "tierra caliente" (30.8\%), en manantiales o en el "monte" (15.4\%). Tal es el caso del berro (Nasturtium officinale), de las verduras de clima cálido como el chipilín (Crotalaria longirostrata) y de diversas especies de hongos como los jonguillos (Agaricus sp.), el kananchay (Lactarius gpo. deliciousus), las orejitas (Auricularia sp., Peziza sp.) y los tashos (Coprinus sp.), considerados como "verduras de monte". Los jefes de familia que participaron en las entrevistas asocian dichas plantas arvenses comestibles con climas cálidos, propios de tierra caliente, y refieren que en tierra fría su crecimiento es escaso. Por esta razón, desde hace al menos cuarenta años, no se acostumbra el fomento de tales verduras dentro del huerto familiar, sino que dichas plantas son toleradas en el traspatio en caso de aparición espontánea. Las verduras silvestres que se cultivan en los huertos familiares observados también pueden extraerse directamente de los espacios abiertos ya mencionados. En estos casos, las madres de familia solicitan a sus maridos o hijos varones que recolecten tales hierbas en la milpa o cuando se trasladan hacia otros lados ya sea durante su trabajo o en una salida específica para su búsqueda. A su regreso, los varones traen consigo alguna verdura que ellos o sus mujeres desean que crezca en casa para integrarla a su traspatio. Si las mujeres requieren alguna especie difícil de cultivar en su huerto, como el berro (Nasturtium officinale), solicitan esta planta a sus esposos o hijos mayores para que vayan a abastecerse directamente al manantial o sitio de colecta de la especie en cuestión. Los paseos familiares y las estancias de trabajo prolongadas en las milpas donde se requiere la participación de la mayoría de los miembros de la familia, también sirven para este fin. Otra forma de abastecimiento de verduras silvestres en el traspatio consiste en solicitar semillas a otros miembros del grupo familiar, vecinos o amistades que cuenten con plantas maduras que hayan conservado para este fin. Las redes sociales se utilizan para el intercambio, adquisición y abastecimiento tanto de semillas, como de las mismas plantas, ya sean enteras o partes vegetativas susceptibles de reproducción asexual, como "codos" o raíces. De esta manera, el flujo de especies entre un sitio de abastecimiento y otro suele ser bidireccional. No sólo se traen al huerto especies procedentes de otros hábitats de colecta, sino que en el huerto familiar también germinan y cultivan temporalmente plantas para trasplantar a parcelas de "tierra caliente", especialmente cuando se trata de especies que al madurar no toleran climas templados o fríos como el de Teopisca. Pese a su importancia como sitio de abastecimiento y a que todas las integrantes del colectivo viven en unidades habitacionales con huerto familiar disponible, se encontró que el 58.3\% (n=7) de las entrevistadas proporciona cuidado y atención cotidiana a este espacio, en comparación con el 41.7\% $(\mathrm{n}=5)$ restante que no lo hace. Sin embargo, este último porcentaje sustituye el papel abastecedor del huerto a través de visitas a los traspatios de la madre o hermanas, el recibimiento de regalos de parte de éstas o la compra de verduras frescas tanto en el mercado local, como en el Tianguis de Comida Sana y Cercana de San Cristóbal de Las Casas. 


\section{Preparación}

Existen diferentes formas de preparación aplicadas a las trece especies reportadas (cuadro 1). Por su sencillez y ausencia de grasas de origen animal al guisar o freír en la Mesoamérica precolombina, la mayor parte de estas técnicas se consideran propias de la cocina prehispánica (Barros y Buenrostro, 2002; De’Angeli y De’Angeli, 2007). El consumo directo sin cocción se reportó en un 30.8\% (n=4) de las especies, el 76.9\% (n=10) se prepara hirviéndolas, el 23\% ( $\mathrm{n}=3)$ se fríen, guisan o consumen en tamales y el $15.4 \%(n=2)$ se cocina con el apoyo de un comal. El cuadro 2 presenta las 33 recetas de cocina documentadas entre las integrantes del Colectivo Mujeres y Maíz de Teopisca que requieren para su elaboración la presencia de al menos una de las especies registradas en el cuadro l. Las verduras silvestres predominantes están reportadas en las recetas incluidas en la figura 1 . La especie con mayor cantidad de apariciones es el chipilín (Crotalaria longirostrata), requerido en al menos seis de los 33 platillos reportados. En orden descendente continúan la mostaza (Brassica juncea), el bledo (Amaranthus hybridus), el berro (Nasturtium officinale), el epazote blanco (Chenopodium ambrosioides), el epazote rojo (Ch. graveolens), el tsuy (Sinclairia discolor) y el mumu (Piper auritum). Exceptuando la mostaza y el berro, todas estas especies se encuentran dentro de su zona de distribución natural (cuadro 1). Debido al origen nativo de las verduras protagónicas y al empleo de técnicas, utensilios y otros ingredientes representativos de la cocina mexicana tradicional, se estima que al menos un 18.2\% ( $\mathrm{n}=6$ ) de las recetas documentadas cuenta con más de tres generaciones de arraigo en los grupos familiares bajo estudio (cuadro 2).

\section{Consumo}

Las especies estudiadas forman parte de la dieta cotidiana de las integrantes del colectivo. La mayoría de los platillos presentados en el cuadro 2 no incluye alimentos que las entrevistadas consideren para ocasiones especiales, como celebraciones religiosas o festividades familiares. Son alimentos comunes en la vida cotidiana de las mujeres, cuyo consumo simboliza humildad o sencillez ya que son alimentos "a la mano", "fáciles de conseguir". En la dieta familiar son habituales las verduras que se incorporan a la olla de frijoles cuando ésta todavía está en el fuego, o bien se sirven a manera de sopa acompañadas de caldo de frijol dentro del mismo plato. Esta forma de consumo ocurre tanto a nivel doméstico, como en los espacios campesinos de trabajo. Durante su almuerzo en la milpa, los agricultores pueden buscar plantas con hojas tiernas de verdolagas (Portulaca oleracea), mumu (Piper auritum), tsuy (Sinclairia discolor), lengua de vaca (Rumex obtusifolius) o chipilín (Crotalaria longirostrata), disponibles in situ para recalentar el caldo de frijol que acompañan de tortilla, chile y limón (cuadro 1).

\section{Discusión y conclusiones}

\section{Diversidad local de verduras silvestres en las prácticas culinarias del Colectivo Mujeres y Maíz de Teopisca}

Brassicaceae, Ateraceae y Chenopodiaceae son las familias taxonómicas mejor representadas, las cuales coinciden con tres de las seis familias que Bye y Linares (2000) agrupan en el $89 \%$ de los quelites o plantas arvenses comestibles conocidos en el México contemporáneo. Aunque la proporción entre especies nativas $(62 \%, \mathrm{n}=5)$ es mayor que la de las introducidas ( $38 \%, \mathrm{n}=5)$, en ambos casos se trata de especies no comerciales destinadas al autoconsumo, cuya ingesta no contemplan los modelos de mercado y alimentación dominantes. Ejemplo de ello es que ninguna de estas verduras se encontró a la venta en el supermercado local durante los meses de trabajo en campo. Por lo tanto, la categoría de "verduras olvidadas" no sólo es aplicable a especies nativas bajo riesgo de ser omitidas dentro de un sistema alimentario dado, sino también a aquellas introducidas que a lo largo del tiempo se incorporaron a dicho sistema. Tal es el caso del berro (Nasturtium officinale), la mostaza (Brassica juncea) o el nabito (Brassica rapa). Lo anterior significa, como propusieron Castro y Balzaretti (2003), 
que el estudio de las transformaciones de la diversidad local de especies comestibles coadyuva al análisis de la relación entre una cultura y la biodiversidad con la que cohabita a lo largo del tiempo.

Acciones y saberes relacionados con la diversidad local de verduras silvestres presentes en las prácticas culinarias del Colectivo Mujeres y Maíz Teopisca

A diferencia de lo reportado por Pardo-Núñez (2004), el concepto local de "verdura" entre las entrevistadas incluye no sólo hierbas comestibles en etapas inmaduras, sino también distintas especies de hongos silvestres recolectados anteriormente en zonas boscosas al comienzo de la estación lluviosa y algunas de las cuales se ofrecen en el mercado local durante la temporada correspondiente. Dado que éste fue un dato derivado del trabajo de campo, las verduras silvestres a las que se refiere el presente estudio no contemplan especies de hongos. Sin embargo, se recomienda que se incluya este grupo de especies como parte de la categoría "verduras" en las investigaciones efectuadas en la región Altos de Chiapas.

Sobresale el papel de los huertos familiares como espacios de confluencia de verduras silvestres, tanto nativas como introducidas, pues el 92.3\% ( $\mathrm{n}=12$ ) de las hierbas se encontró en estos sitios, además de en otros hábitats. De lo anterior se concluye que existe un flujo de especies bidireccional entre el huerto y el resto de los espacios locales de abastecimiento, por lo que este sitio estimula la interacción con otros hábitats. Estos resultados concuerdan con los de Moreno-Black et al. (1996), quienes al estudiar los huertos familiares del noreste de Tailandia encontraron que en éstos conviven tanto especies cultivadas como silvestres, nativas e introducidas, de las cuales las de usos alimentarios fueron las más representativas. Moreno-Black et al. (1996) afirman que las mujeres juegan un papel notable al seleccionar especies vegetales locales para trasplante y manejo dentro de sus huertos, lo que aumenta las posibilidades de que tales especies sobrevivan a través del tiempo y conformen un refugio para la preservación de variedades locales.
Adicionalmente, las relaciones sociales tanto a nivel familiar y doméstico, como a través de vecinos y entre los propios grupos familiares que integran el Colectivo Mujeres y Maíz, favorecen el mencionado flujo de especies entre el huerto familiar y el resto de espacios de abastecimiento. Así, el huerto familiar posee un carácter dinámico y abierto, tanto espacial como socioculturalmente, pues en él se traza una red de relaciones sociales y espaciales que posibilita a las mujeres del Colectivo el suministro cotidiano de alimentos provenientes de la biodiversidad local $y$, por ende, el mantenimiento de prácticas y saberes relacionados con ésta, aun cuando el huerto familiar no reciba la misma atención o manejo que en otras áreas del sureste mexicano. Esto concuerda con lo expuesto por Moreno-Black et al. (1996), en que los huertos familiares no sólo son expresión de la interacción cotidiana de las mujeres y sus familias con los procesos ambientales de su comunidad, sino que también se trata de espacios que proporcionan estabilidad ante los cambios socioeconómicos de la actualidad.

No obstante la importancia de los huertos familiares como espacios de crecimiento, abastecimiento y preservación de especies locales silvestres, su mención es poco frecuente en la literatura especializada sobre quelites o verduras silvestres en México (Bye y Linares, 2000; Mera et al., 2005; Boege, 2008; Resina, 2010). Por este motivo es imperioso que, al explorar el tema de verduras olvidadas en México, además de realizar estudios dirigidos al sistema milpa, se enfatice el papel que juegan los huertos familiares.

En nuestro país, el huerto familiar es de especial relevancia en las zonas donde residen mayoritariamente indígenas (Mariaca et al., 2010), como Los Altos de Chiapas. Sin embargo, sobresale el hecho de que el $41.7 \%(n=5)$ de las mujeres entrevistadas no proporciona a este espacio atención cotidiana, de modo que el huerto familiar en estos casos no posee la misma trascendencia que en otras zonas del sureste mexicano como abastecedor de verduras silvestres ni sitio de enseñanza, aprendizaje o experimentación en cuanto a las prácticas alimentarias relacionadas con dichas especies. Lo anterior podría explicarse en parte por las condiciones climáticas 
propias de Teopisca, las cuales son poco favorables para el crecimiento de diversas plantas, las cuales, según los entrevistados, prosperan de mejor manera en "tierra caliente". Otra explicación puede ser que el ritmo de trabajo de dichas mujeres no les permite disponer del tiempo necesario para atender el huerto familiar de manera constante, ya que dentro del colectivo existen mujeres que, además de atender sus labores domésticas y familiares, elaboran alimentos para vender en la ciudad de San Cristóbal de Las Casas varias veces por semana. También hay integrantes del colectivo que prefieren la cría de gallinas en lugar del cuidado y cultivo de hierbas comestibles. Lo anterior se debe a que, aunque dichos animales impiden el crecimiento de herbáceas, son susceptibles de venta en casos de emergencia económica. Sin embargo, hierbas como el tsuy (Sinclairia discolo), la chicoria (Sonchus oleraceus) o la verdolaga (Portulaca oleracea), que crecen espontáneamente en macetas elevadas y áreas fuera del alcance de aves de corral, son susceptibles de aprovechamiento sin necesidad de cuidados especiales. En contraste, en los huertos familiares de dos integrantes del colectivo se observó una mayor diversidad de hierbas comestibles y un mejor cuidado de las mismas. Ambos casos coinciden en que el principal lugar de venta de tales mujeres es el Tianguis de Comida Sana y Cercana en San Cristóbal de Las Casas. Este último es un espacio comprometido con los mismos principios de apoyo a la producción local de alimentos libres de insumos químicos y cultivo de variedades locales bajo los que trabaja el Colectivo Mujeres y Maíz (RPCRCSC, 2012). Así, es probable que iniciativas como el tianguis estén propiciando una mayor atención hacia las verduras de traspatio, muchas de ellas silvestres, por parte de las mujeres del colectivo.

De las trece especies de verduras silvestres encontradas, el chipilín (Crotalaria longirostrata) es la hierba con mayor frecuencia de uso dentro de las prácticas culinarias de las mujeres del colectivo (figura 1). Según Muñoz (2000), esta verdura es exclusiva del sureste del país, "donde se utiliza como quelite". La relevancia culinaria de esta hierba también sobresale en recetarios de cocina chiapaneca donde, de doce recetas con empleo de hierbas comestibles, el chipilín aparece en siete (58.3\%) (CONACULTA, 2000). La importancia culinaria del resto de las especies predominantes en las recetas encontradas también ha sido ampliamente documentada (Bye y Linares, 2000; Muñoz, 2000; PardoNúñez, 2004).

Se estima que las mujeres que encabezan los grupos familiares en estudio poseen saberes culinarios asociados a verduras locales silvestres que les fueron transferidos por sus madres y abuelas y que aún ponen en práctica cotidianamente, por lo que se amplía la posibilidad de continuar la transmisión de éstos por línea materna y al interior del colectivo. Lo anterior según: 1) las referencias familiares expresadas en las entrevistas, 2) la antigüedad calculada para las recetas registradas (cuadro 2) y 3) la continuidad de otras prácticas asociadas como el consumo de caldo de frijoles cocidos con verduras recolectadas en sus hábitat naturales.

En cuanto al olvido de ciertas verduras silvestres, diversos autores (Bye y Linares, 2000; De Garine y Vargas, 2006; De'Angeli y De'Angeli, 2007) sugieren que especies más comerciales y de amplia distribución, como las acelgas, berros o espinacas, sustituyen fácilmente ingredientes locales como los quelites. No obstante, para las mujeres del colectivo, el consumo de verduras locales está relacionado con el reconocimiento al trabajo y los conocimientos de los campesinos; además, con una buena salud y nutrición, así como con la autonomía y autosuficiencia alimentarias. De este modo, el desplazamiento de las verduras silvestres de la cultura alimentaria local implica más que su sola sustitución pues, al perderse éstas, desaparecen también conocimientos sobre la biodiversidad local, una forma de alimentación adaptada a las condiciones climáticas, culturales y ecológicas particulares de la comunidad en cuestión y la capacidad para el autoabastecimiento de alimentos que fortalezcan a sus consumidores tanto fisiológica como culturalmente. En relación con lo anterior, Escobar-Ibáñez (2010: 27) sostiene que "a mayor reflejo del ecosistema en una cultura alimentaria, hay una mayor adaptación al mismo", de modo que la gastronomía local expresa la adaptación cultural de una sociedad hacia los ecosistemas en los que se inserta. 
En ese sentido, es necesario continuar con labores de difusión de los conocimientos culinarios encontrados, así como apoyar labores de valoración de variedades locales de maíz y la biodiversidad a él asociada, como las que realizan las mujeres del Colectivo Mujeres y Maíz. Las acciones cotidianas y sistemáticas generan un impacto considerable, por lo que se sugiere la constante incorporación de alimentos locales en la comida cotidiana de cada persona, la preferencia por el consumo de estos productos en los espacios de venta correspondientes y el fomento de verduras locales tanto en huertos urbanos, como en traspatios convencionales. Proyectos como el que documentan Linares y otros (2006), en el que se combinan estrategias educativas con programas de difusión, actualización y capacitación, basados en encuestas diagnósticas a sectores poblacionales específicos, han obtenido resultados favorables.

A partir de este trabajo, las mujeres del Colectivo Mujeres y Maíz de Teopisca dispondrán de información útil para tomar decisiones sobre las verduras locales que desean conservar o fomentar tanto en los espacios locales de abastecimiento a los que tienen acceso, como en su dieta y quehacer cotidianos de acuerdo con los gustos y necesidades alimentarias de sus familias y de sus consumidores. Estas mujeres tienen, además, la posibilidad de compartir sus saberes y prácticas culturales, asociados a verduras locales, con los habitantes de Teopisca y San Cristóbal de Las Casas, y con ello pueden ayudar a revertir el proceso de desvinculación urbana con la diversidad de alimentos locales y ecosistemas que los rodean.

\section{Notas}

${ }^{1}$ El término "monte" se usa en referencia a terrenos elevados donde suele existir vegetación boscosa. Las verduras cuyo nombre común se acompaña de esta palabra corresponden generalmente a especies silvestres o con escaso grado de domesticación. Otra acepción local del término "monte" corresponde a herbáceas de origen silvestre presentes en terrenos de cultivo a pesar de no haber sidocultivadas ahí. Los "montes" que tienen usos alimentarios para el ser humano también caen dentro de la categoría local de "verduras".
${ }^{2}$ En dicho trabajo se establece que la categoría "verdura" se utiliza en esta zona para designar a las hojas verdes y flores de hierbas o arbustos, cultivados o no, que fungen como ingredientes principales de un guiso y representan el plato principal de la comida o cena del día.el plato principal de la comida o cena del día.

\section{Referencias bibliográficas}

Altieri, Miguel y Clara I. Nicholls, (2005) Agroecology and the Search for a Truly Sustainable Agriculture. México, PNUMA.

Bailey, Liberty Hyde, (1966) Manual of Cultivated Plants Most Commonly Grown in Continental United States and Canada. Nueva York, The MacMilan Company.

Barros, Cristina y Marco Buenrostro, (2002) "Cocina prehispánica. Continuidad cultural. Recetario” en Arqueología Mexicana. Edición especial 12. México, Raíces/INAH.

Bélanger Julie y Timothy Johs, (2008) "Biological Diversity, Dietary Diversity, and Eye Health in Developing Country Populations: Establishing the Evidence-base" en EcoHealth. Núm. 5, pp. 244-256.

Boege Schmidt, Eckart, (2008) "Las regiones bioculturales según los inventarios de la diversidad biológica domesticada y semidomesticada" en Eckart Boege Schmidt (coord.) El patrimonio biocultural de los pueblos indígenas de México. Hacia la conservación in situ de la biodiversidad y agrodiversidad en los territorios indígenas. México, INAH/ CDI, pp. 158-229.

Bye, Robert y Edelmira Linares, (2000) “Los quelites, plantas comestibles de México: una reflexión sobre intercambio cultural" en Biodiversitas. Vol. 31, pp. 11-14.

Bye, Robert, Miguel et al., (2008) "Proyecto "Milpa". Etnobotánica de quelites. México, Genetic Resources Conservation Program. Disponible en: http://www. grcp.ucdavis.edu/milpa/MCK996/QUEL96.HTM [consultado el 7 de enero de 2012].

Calderón de Rzedowski, Graciela y Jerzy Rzedowski, (2001) Flora fanerogámica del Valle de México. México, CONABIO/Instituto de Ecología, A.C.

Castro, Elba y Karim Balzaretti, (2003) "Pérdida de diversidad biológica y cambio de cultura alimentaria. Lectura y aportaciones de la educación ambiental" 
en Faustino Moreno Ceja y María de Rocío Zumaya Leal (comps.), Educación, salud y medio ambiente. México, Universidad de Guadalajara, pp. 151-174.

CEIEG. Comité Estatal de Información Estadística y Geográfica de Chiapas, (2010) Atlas de Chiapas. Tuxtla Gutiérrez, Chiapas, México, CEIEG. Disponible en: http://www.ceieg.chiapas.gob.mx/home/wp-content/ uploads/downloads/2011/04/atlas2010/Atlas2010.swf [consultado el 27 de octubre de 2012].

CONACULTA. Consejo Nacional para la Cultura y las Artes, (2000) La cocina familiar en el estado de Chiapas. México, CONACULTA/Océano.

Crosby, Alfred W., (2003) "La fusión de dos comidas" en Janet Long (coord.), Conquista y comida: consecuencias del encuentro entre dos mundos. México, UNAM, pp. 131-144.

De’Angeli, Alicia Gironella y Giorgio De'Angeli, (2007) Larousse de la cocina mexicana. México, Larousse.

De Garine, Igor y Luis Alberto Vargas, (2006) "Introducción a las investigaciones antropológicas sobre alimentación y nutrición" en Cuadernos de Nutrición. Núm. 20, pp. 21-28.

Diamond, Jared, (2007) Armas, gérmenes y acero. México, DeBolsillo.

Díaz Méndez Cecilia y Cristóbal Gómez Benito, (2001) "Del consumo alimentario a la sociología de la alimentación", en Distribucióny consumo. Núm. 60, pp. 5-24.

Dillon, Michael O., (2001) "Sinclaria", en W.D. Stevens, Carmen Ulloa Ulloa, Amy Pool y Olga Martha Montiel (eds.), Flora de Nicaragua. St. Louis, Missouri, Missouri Botanical Garden Press, p. 369.

Escobar-Ibañez, Mariana, (2010) Saberes ambientales de la comunidad rarámuri ba'winocachi a partir de la recolección de plantas silvestres comestibles. Tesis de licenciatura. Universidad de Guadalajara, Guadalajara, México.

Fernández-Armesto, Felipe, (2004) Historia de la comida: alimentos, cocina y civilización. España, Tusquets.

Flores Reyes, Lorena, (2012) Intercambio de plantas, semillas y conocimientos del huerto entre grupos culturales urbanos de San Cristóbal de las Casas, Chiapas. Tesis de maestría. El Colegio de la Frontera Sur, México.

Gobierno Municipal de Teopisca, (1984) Teopisca diagnóstico municipal. México, Gobierno del Estado de Chiapas.
González Arce, Romano, (2008) "De flores, brotes y palmitos: alimentos olvidados" en Agronomía Costarricense. Núm. 32, pp. 183-192.

González de Cosío, Manuel, (1984) Especies vegetales de importancia económica en México. México, Porrúa.

Heiser, Charles, (1985) "Chenopods: From Weeds to the Halls of Moctezuma" en Charles Heiser, Of Plants and People. Oklahoma, University of Oklahoma Press, pp. 82-99.

INAFED. Instituto Nacional para el Federalismo y el Desarrollo Municipal, (2005) "Estado de Chiapas. Teopisca" en Enciclopedia de municipios de México. México, Gobierno del Estado de Chiapas. Disponible en: http://www.e-local.gob.mx/work/templates/enciclo/ chiapas/municipios/07094a.htm [consultado el 10 de noviembre de 2011].

INEGI. Instituto Nacional de Estadística y Geografía, (2010) Catálogo de claves de entidades federativas, municipios y localidades. México, INEGI. Disponible en: http://mapserver.inegi.org.mx/mgn2k/?c=646\&ss=est [consultado el 10 de octubre de 2011].

Langreo Navarro, Alicia, (2008) "El sistema alimentario mundial. Principales tendencias y efectos sobre los sistemas alimentarios locales" en Distribución y Consumo. Núm. 100, pp. 258-274.

Linares, Edelmira, Teodolina Balcázar y Robert Bye, (2006) Las verduras mexicanas silvestres los "quelites". Antiguos compañeros hoy casi olvidados. México, Botanic Gardens Conservation International. Disponible en: http:// www.bgci.org/education/article/249/[consultado el 13 de enero de 2012].

Loftas, Tony, (1995) Dimentiosn of Need. An Atlas of Food and Agriculture. Roma, FAO. Disponible en: http://www.fao. org/docrep/U8480E/U8480E00.htm [consultado el 13 de noviembre de 2011].

Logan, Michael H., Kimberly D. Gwinn, Tina Richey, Beth Maney y Charles T. Faulkner, (2004) "An Empirical Assessment of Epazote (Chenopodium ambrosioides) as a Flavoring Agent in Cooked Beans" en Journal of Ethnobiology. Vol. 1, núm. 24, pp. 1-12.

Mariaca, Ramón, Alba González-Jácome y Luis Manuel Arias Reyes, (2010) El huerto mayayucatecoen el siglo XVI. México, Universidad Iberoamericana/El Colegio de la Frontera Sur/Cinvestav-Unidad Mérida/UIMQroo. 
McDade, T.W., V. Reyes-García, P. Blackinton, S. Tanner, T. Huancay R. Leonard, (2007) “Ethnobotanical Knowledge is Associated with Indices of Child Health in the Bolivian Amazon" en Proceedings of the National Academy of Sciences. Núm. 15, pp. 6134-6139.

Meléndez Torres, Juana María y Gloria María Cañez de la Fuente, (2010) "La cocina tradicional regional como elemento de identidad y desarrollo local. El caso de San Pedro El Saucito, Sonora, México" en Estudios Sociales. Número especial, pp. 184-204.

Mera Ovando, L.M. et al., (2005) "De quelites me como un taco" en Ciencias. Vol. 77, pp. 36-38.

Moreno-Black, Geraldine, Prapimporn Somnasang y Sompong Thamathawan, (1996) "Cultivating Continuity and Creating Change: Women's Home Garden Practices in Northeastern Thailand" en Agriculture and Human Values. Vol. 3, pp. 3-1l.

Muñoz Zurita, Ricardo, (2000) Diccionario enciclopédico de la gastronomíamexicana. México, Clío.

Mujeres y Maíz, (2010) Mujeres y maíz criollo. Blog en línea. México. Disponible en: http://mujeresymaiz.blogspot. com/p/contactenoscontact-us.html [consultado el 9 de enero de 2012].

Nájera Castellanos, Antonio de Jesús y Guadalupe del Carmen Álvarez Gordillo, (2010) "Del posol a la Coca Cola: cambios en las prácticas alimentarias en dos comunidades tojolabales" en LiminaR. Estudios Sociales y Humanísticos. Núm. 1, pp. 173- 190.

New Agriculturalist, (2008) Making More of Undervalued Crops. Reino Unido, WRENmedia. Disponible en: http://www.newag.info/en/pov/views.php?a=426 [consultado el 9 de enero de 2012].

Pardo-Núñez, Joaliné, (2004) La verdura como categoría local: consideraciones socioculturales de su proceso comercial en Los Altos de Chiapas. Tesis de maestría, México, El Colegio de la Frontera Sur.

PESA-Centroamérica. Proyecto Estratégico para la Seguridad alimentaria de la Organización de las naciones Unidas para la Agricultura y la Alimentación en Centroamérica, (2010) Seguridad alimentaria y nutricional. Conceptos básicos. Costa Rica, FAO.

Ramírez-Salinas Concepción y Adriana Elena CastroRamírez, (2011) 'Los 'Montes', conocimiento tradicional campesino sobre las arvenses de la milpa en Teopisca y
Amatenango del Valle, Chiapas", en León Enrique Ávila Romero (coord.), Desarrollo sustentable, interculturalidad y vinculación comunitaria. México, Universidad Intercultural de Chiapas, pp. 95-117.

RPCRCSC. Red de Productores y Consumidores Responsables Comida Sana y Cercana, (2012) Red Comida Sanay Cercana, Blog en línea, México. Disponible en: http:// redcomidasanaycercana.codigosur.net/ [consultado el 6 de diciembre de2012].

Resina, (2010) Red quelites. México, Sistema Nacional de Recursos Filogenéticos para la alimentación y la Agricultura. Disponible en: www.resina.org.mx [consultado el 5 de septiembre de 2011].

Rodríguez Gómez, Guadalupe, (2007) “Imaginar la soberanía alimentaria y las oportunidades para las agriculturas del México globalizado" en Desacatos. Núm. 25, pp. 101-114.

Rosset, Peter M., (2007) "Mirando hacia el futuro: La reforma agraria y la soberanía alimentaria” en Revista Internacional de Ciencias Sociales. Núm. 26, pp. 167-182.

Santamarina Campos, Beatriz, (2008) "Antropología y medio ambiente. Revisión de una tradición y nuevas perspectivas de análisis en la problemática ecológica" en Revista de Antropología Iberoamericana. Núm. 2, pp. 144-184.

Szabó, Pétery Radim Hédl, (2011) “Advancing the Integration of History and Ecology of Conservation" en Conservation Biology. Vol. 4, núm. 25, pp. 680-687.

Toledo, Víctor Manuel, Julia Carabias, Cristina Mapes y Carlos Toledo, (1985) Ecología y autosuficiencia alimentaria. México, Siglo XXI.

Universidad Autónoma Chapingo, (2001) Base de información sobre especies con potencial de abonos verdes y cultivos de cobertura. México, Centro Universitario de Educación VirtualUniversidad Autónoma Chapingo. Disponible en: http:// www.virtual.chapingo.mx/dona/paginaIntAgronomia/ abonoverde2.pdf_[consultado el 16 de agosto de 2012].

Vanden Eynden, VeerleyEduardoCueva,(2008) “Lasplantas en la alimentación” en Lucía de la Torre, Hugo Navarrete, P. Muriel M., Manuel J. Macía y Henrik Balslev (eds.) Enciclopedia de las plantasútiles del Ecuador. Ecuador, Quito \& Aarhus/Herbario QCA \& Herbario AAU, pp. 62-66.

Vibrans, Heike, (2012) Malezas de México, México, CONABIO. Disponible en: http://www.conabio.gob. $\mathrm{mx} / \mathrm{malezasdemexico/2inicio/home-malezas-mexico.}$ htm [consultado el 24 de abril de 2013]. 


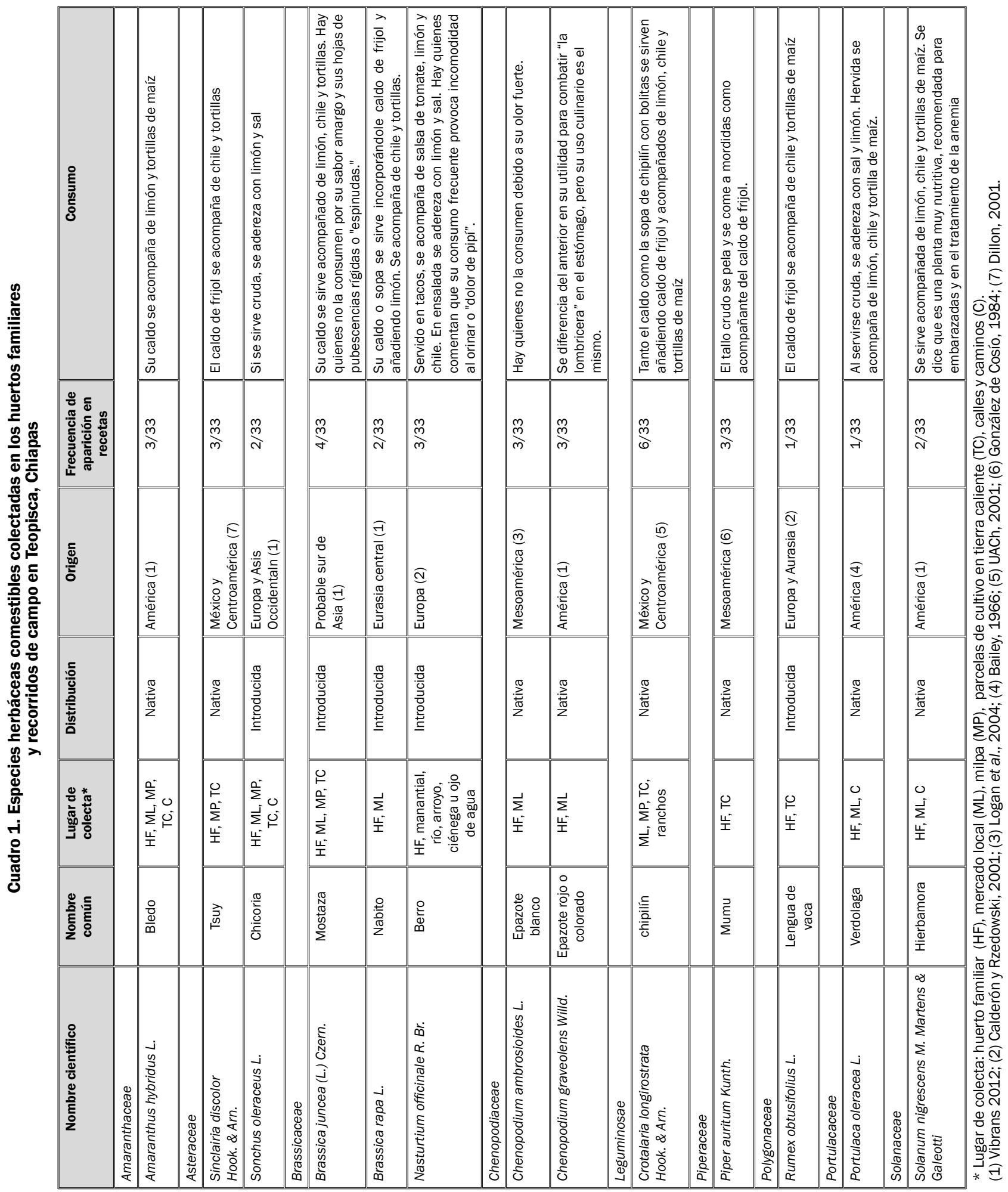




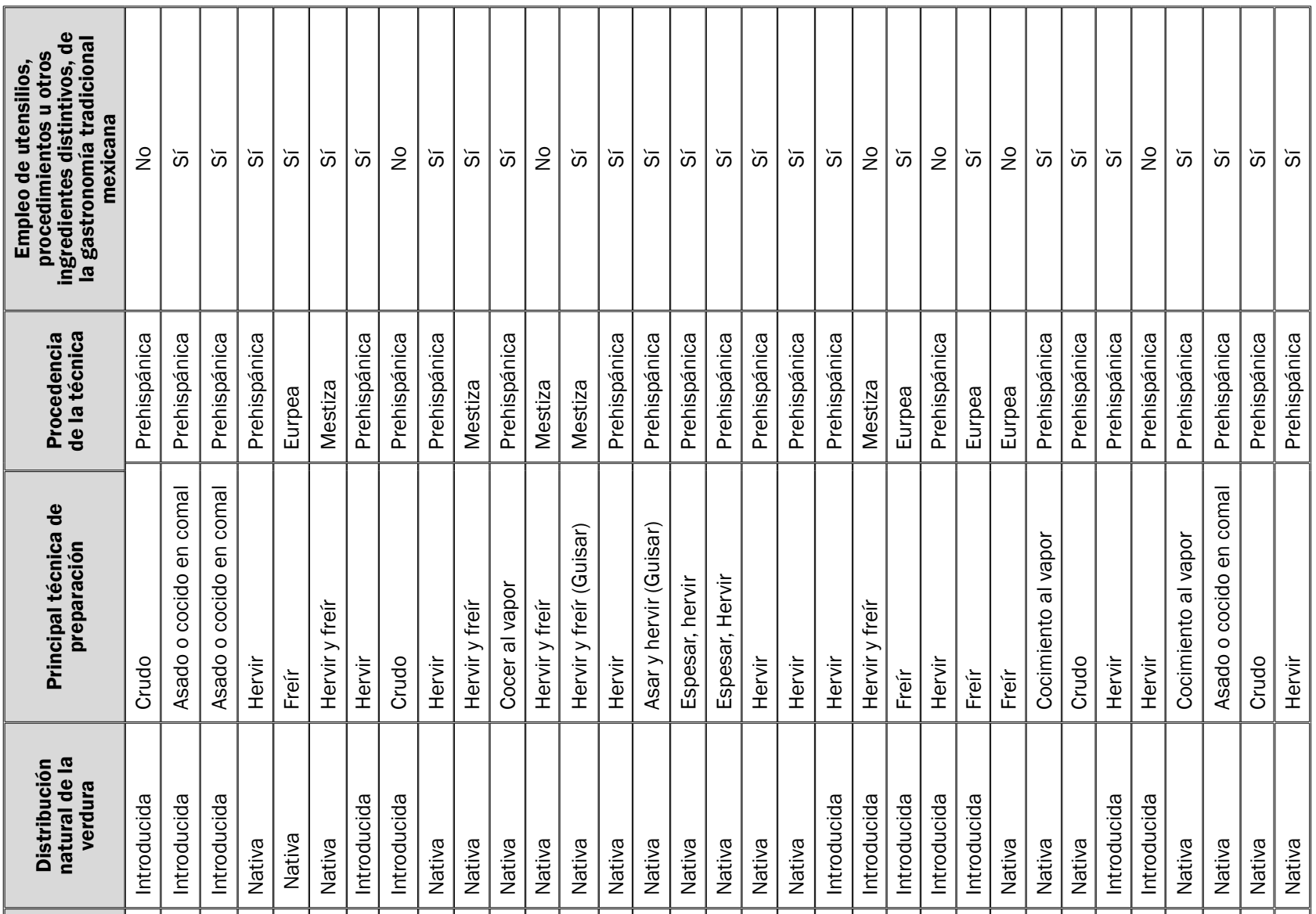

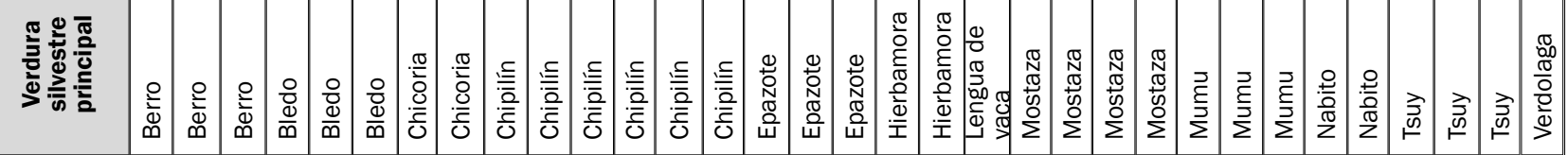

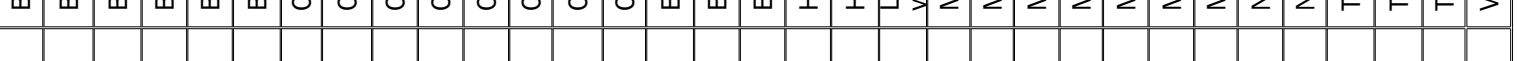

일

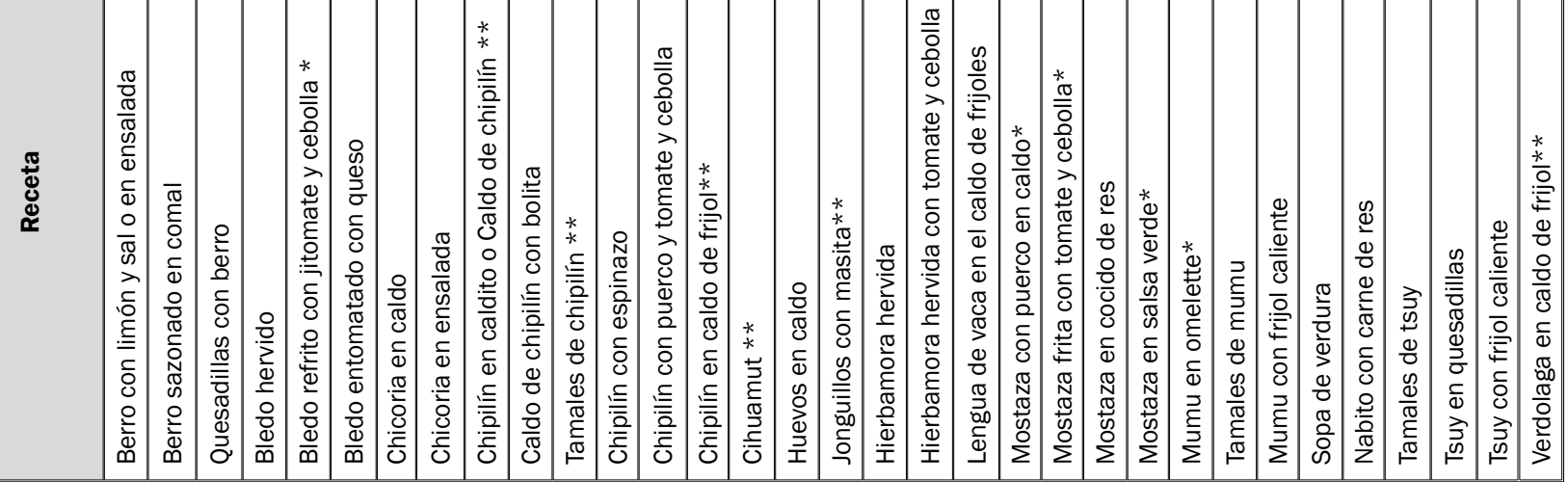

$\stackrel{\frac{0}{2}}{\frac{0}{2}}$

这

要需

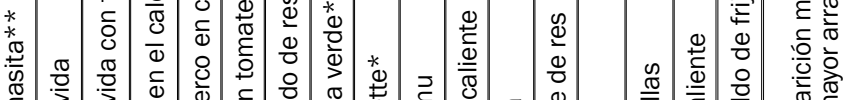

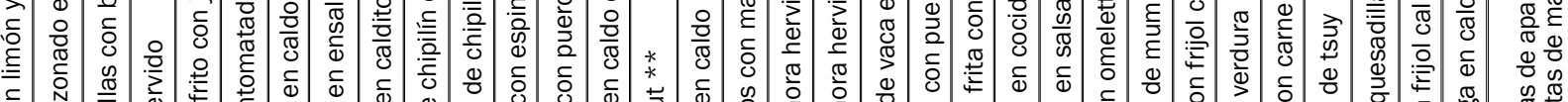

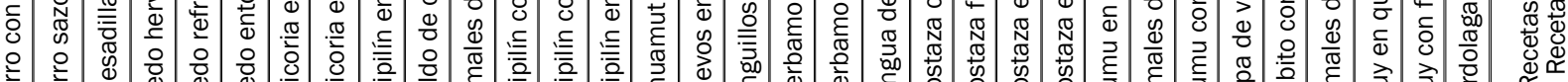

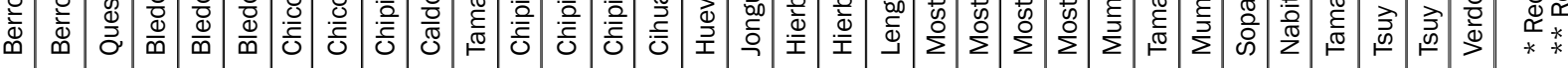


Mapa 1. Ubicación de la localidad de Teopisca, Chiapas

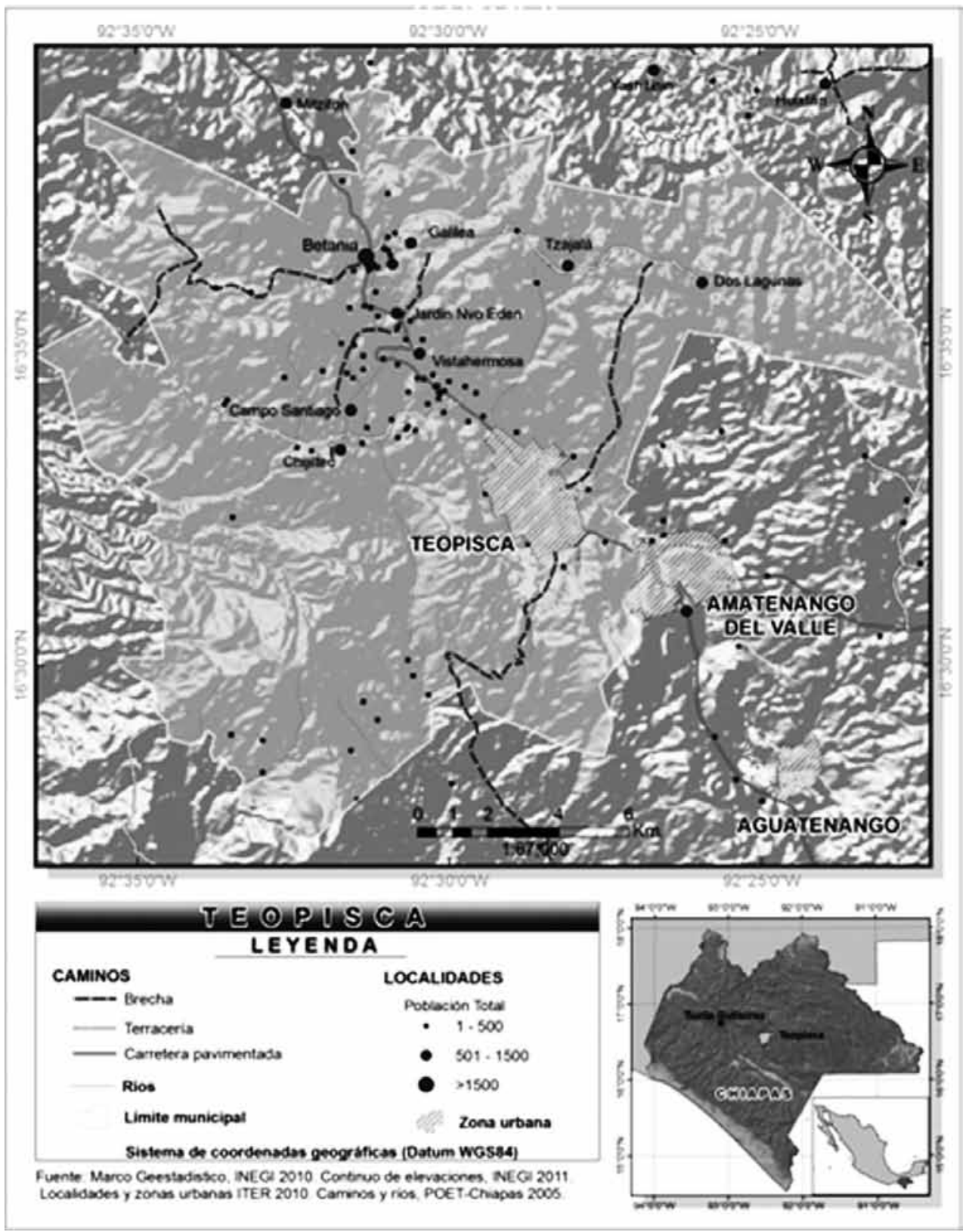

Fuente: diseño cartográfico, David Uribe. 
Figura 1. Número de recetas en las que participa cada verdura silvestre estudiada

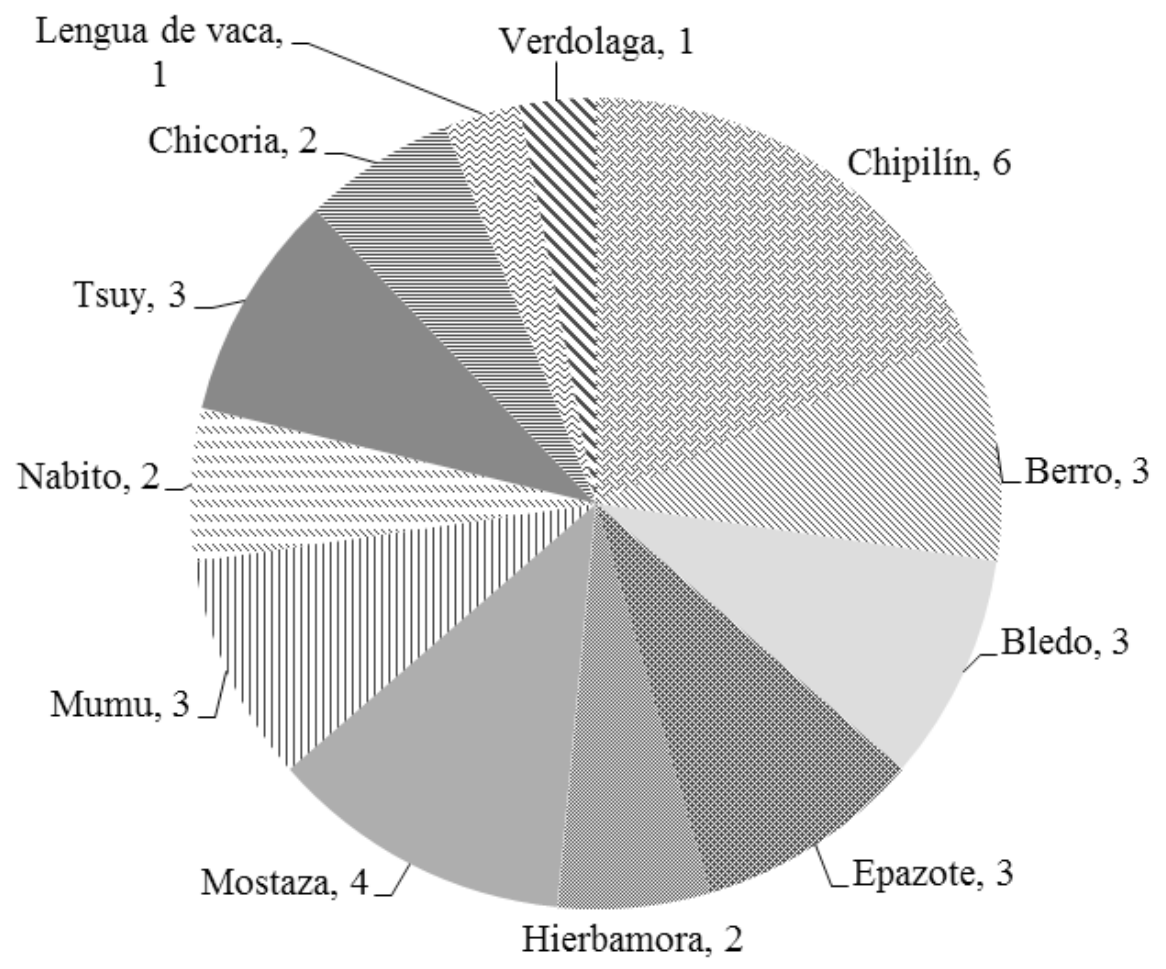

Fuente: elaboración propia. 